ACCEPTED FOR PUblication IN ApJ

Preprint typeset using IATEX style emulateapj v. 4/12/04

\title{
CHEMICAL SIGNATURE INDICATING A LACK OF MASSIVE STARS IN DWARF GALAXIES
}

\author{
Takuji Tsujimoto \\ National Astronomical Observatory of Japan, Mitaka-shi, Tokyo 181-8588, Japan; taku.tsujimoto@nao.ac.jp \\ Accepted for publication in ApJ
}

\begin{abstract}
Growing evidence supports an unusual elemental feature appearing in nearby dwarf galaxies, especially dwarf spheroidals (dSphs), indicating a key process of galaxy evolution that is different from that of the Galaxy. In addition to the well-known deficiency of $\alpha$-elements in dSphs, recent observations have clearly shown that $s$-process elements $(\mathrm{Ba})$ are significantly enhanced relative to $\mathrm{Fe}, \alpha$-, and $r$-process elements. This enhancement occurs in some dSphs as well as in the Large Magellanic Cloud, but is unseen in the Galaxy. Here we report that this feature is evidence of the lack of very massive stars $\left(\gtrsim 25 M_{\odot}\right)$ as predicted in the low star formation rate environment, and we conclude that the unique elemental feature of dwarf galaxies including a low- $\alpha / \mathrm{Fe}$ ratio in some low-metallicity stars is, at least in some part, characterized by a different form of the initial mass function. We present a detailed model for the Fornax dSph galaxy and discuss its complex chemical enrichment history together with the nucleosynthesis site of the light $s$-process element Y.
\end{abstract}

Subject headings: stars: abundances — galaxies: abundances — galaxies: dwarf — galaxies: evolution

\section{INTRODUCTION}

Nearby dwarf galaxies provide an excellent test bed for understanding how the Local Universe has evolved with cosmic time. This has been made feasible by 8 -m-class telescopes, which can access individual stars in nearby dwarf spheroidal (dSph) galaxies (Tolstov et al. 2009) as well as in the Magellanic Clouds (Hill 2004). The detailed abundance data accumulated to date from a significant sample of stars enables us to chemically tag what occurred in these dwarf galaxies based on our knowledge of Galactic archaeology (e.g., Freeman \& Bland-Hawthorn 2002).

A well-known chemical feature observed in dSphs is a lower $\alpha / F e$ ratio compared with that in the Galactic halo stars (e.g., Shetrone et al. 2001; Venn et al. 2004). Recent studies found the same level of $[\alpha / \mathrm{Fe}]$ in stars close to the lowest metallicity (e.g., Frebel et al. 2010a) and a knee in the $[\alpha / \mathrm{Fe}]$ vs. $[\mathrm{Fe} / \mathrm{H}]$ diagram (e.g., Cohen \& Huang 2009); these studies seem to support that the contribution of Fe from type Ia supernovae ( $\mathrm{SNe}$ Ia), starting from a very low metallicity $([\mathrm{Fe} / \mathrm{H}] \ll-1)$ results in the observed low- $[\mathrm{Fe} / \mathrm{H}]$, low- $[\alpha / \mathrm{Fe}]$ stars (e.g., Lanfranchi \& Matteucci 2003; Kirby et al. 2011). However, no clear signature of an SN Ia enrichment for other elements, such as $\mathrm{Mn} / \mathrm{Fe}$ and $n$-capture/Fe, casts doubt on this scheme (Tsuijimoto 2006). Moreover, a low- $\alpha / \mathrm{Fe}$ ratio has been detected for very low-metallicity stars (Aoki et al. 2009), and was also found in the Large Magellanic Cloud (LMC) (Pompéia et al. 2008). This observational fact seems to conflict with the presence of lowmetallicity stars exhibiting high $[\alpha / \mathrm{Fe}]$ in some dSphs (Koch et al. 2008; Venn \& Hill 2008; Frebel et al. 2010b; Letarte et al. 2010), and clear answers to this problem continue to elude us.

In contrast, the abundance of $n$-capture elements, $\mathrm{Ba}$ and La, are enhanced in some dSphs. First, we noticed that $\mathrm{Ba}(\mathrm{La})$ is enhanced relative to the $r$-process element, Eu, by examining an ensemble of data from six dSphs (Venn et al. 2004, references therein). Theoretically, the high $[\mathrm{Ba} / \mathrm{Eu}]$ ratio can be explained with a strong galactic wind model, in which the $r$-process elements from type II SNe (SNe II) are no longer produced after the onset of the winds (Lanfranchi et al. 2008). This issue was remarkably furthered by the abundant data from a high resolution VLT study for the Fornax (Fnx) dSph (Letarte et al. 2010). This study revealed a clear increase in $\mathrm{Ba}$ and $\mathrm{La}$, not only in comparison with $\mathrm{Eu}$ but also with $\mathrm{Fe}$ or $\alpha$-elements in accordance with an increasing $\mathrm{Fe} / \mathrm{H}$ during late evolution. This feature contrasts with that of the Galaxy and disagrees with current model predictions (Lanfranchi et al. 2008). A similar feature is seen in some other dSphs, for example in the Sagitarrius dSph (Sbordone et al. 2007), and clearly in the LMC (Pompéia et al. 2008).

Based on an idea that invoking the initial mass function (IMF) variations may be one of the solutions to explain chemical abundance patterns (e.g., Pagel 1997), we present a new mechanism underlying the observed trends between $\mathrm{Ba}, \mathrm{Eu}$, and $\mathrm{Fe}$ in some dwarf galaxies, with a focus on the Fnx dSph. Our claim is that star formations lacking very massive stars, such as $\gtrsim 25 M_{\odot}$, imprint an unusual $s$-process enhancement, which is observed in stellar abundance. The suppression of the formation of massive stars in low surfacebrightness galaxies is implied from the observed flux ratio of $\mathrm{H} \alpha$ to the far ultraviolet (Meurer et al. 2009). In addition, from a theoretical aspect, recent work suggests that a high mass end $m_{\mathrm{u}}$ of the IMF depends on the mass of the star clusters, and thus on the physical properties of the galaxies where the clusters have formed (Kroupa \& Weidner 2003; Weidner \& Kroupa 2005; Pflamm-Altenburg \& Kroupa 2008). These studies claim that $m_{\mathrm{u}}$ would be lower in the low density environment of dwarf galaxies, in which the formation of massive star clusters is suppressed. Their model predictions have been shown to be consistent with the observed trend for the $\mathrm{H} \alpha$-to-FUV flux ratio (Lee et al. 2009). It is wothwhile to note that, in the Fnx dSph case, the approximate star formation rate (SFR) of $3 \times 10^{-3} M_{\odot} \mathrm{yr}^{-1}$ (Coleman \& de Jong 2008) is predicted to be $m_{u}=20-40$ 
$M_{\odot}$, while galaxies with a high SFR of $\gtrsim 1 M_{\odot} \mathrm{yr}^{-1}$ possess $m_{u}=150 M_{\odot}$ (Weidner \& Kroupa 2005).

This proposed scenario must be reconciled with the framework based on the star formation history, as revealed by extensive surveys for the Fnx dSph (Battaglia et al. 2006; Coleman \& de Jong 2008). We show that this task leads to a grand scheme of chemical evolution for the Fnx dSph, in which a simple model is not applicable, but a complex view provides a deeper understanding of its elemental feature.

\section{MECHANISM OF AN ENHANCED $S$-PROCESS ABUNDANCE \\ 2.1. Abundance patterns and nucleosynthesis}

A comparison of the Ba-Eu-Fe abundance trends between the Fnx dSph and the Galaxy is shown in Figure 1. Information on an early phase $([\mathrm{Fe} / \mathrm{H}] \sim-2.5)$ for the $\mathrm{Fnx}$ $\mathrm{dSph}$ is provided by the abundance of globular clusters (GCs) (Letarte et al. 2006), which must be equivalent to that of interstellar matter at that time. On the upper panel, we see a sharply increasing $[\mathrm{Ba} / \mathrm{Fe}]$ (roughly $0 \rightarrow 1$ ) from $[\mathrm{Fe} / \mathrm{H}] \sim-1$ until $[\mathrm{Fe} / \mathrm{H}] \sim-0.5$. In contrast, in the Galaxy case this value is broadly constant at the level of $[\mathrm{Ba} / \mathrm{Fe}] \sim 0$. On the other hand, for $[\mathrm{Fe} / \mathrm{H}]<-1$, the $[\mathrm{Ba} / \mathrm{Fe}]$ ratio for the dSph remains nearly constant, and is on average higher than that of the Galaxy at $[\mathrm{Fe} / \mathrm{H}] \sim$ 2.5. These two features of the Fnx dSph, i.e., a sharp increase at a late stage and an initially high ratio, can be understood from the theoretical aspect described in the following.

The key point to highlight is that the sites of nucleosynthesis among the $r_{-}, s$-process elements and $\mathrm{Fe}$ are different from each other. Note that $\mathrm{Ba}$ is synthesized through both $r$ - and $s$-processes. While $\mathrm{Fe}$ is synthesized and ejected in all SNe II with masses $\geq 10 M_{\odot}$, it is believed that the site of $r$-process elements is confined to the limited SNe II that are inclined to be less massive. The theoretical interpretation of abundance data on very metalpoor stars implies that the mass range for the $r$-process is 8-10 $M_{\odot}$ (Mathews et al. 1992; Ishimaru \& Wanajo 1999) as identified with the collapsing $\mathrm{O}-\mathrm{Ne}-\mathrm{Mg}$ core (Wheeler et al. 1998), 20-25 $M_{\odot}$ (Tsuijimoto et al. 2000), or 12-30 $M_{\odot}$ (Cescutti et al. 2006) with heavy weighing on lower mass progenitors. On the other hand, the main $s$-process operates in AGB stars with a mass of 1.5-3 $M_{\odot}$ (Busso et al. 2001), that might extend to the more massive hot AGB $\left(\sim 6 M_{\odot}\right)$ (e.g., Goriely \& Siess 2004).

Suppose that the Fnx dSph possesses the IMF with the high-mass end truncated around 25-30 $M_{\odot}$. Such a truncated IMF is motivated by a theoretical background (Weidner \& Kroupa 2005) and also implied from the abundance of some metal-poor damped Lyman- $\alpha$ systems (Tsuijimoto \& Bekki 2011). The effect of its introduction is a reduction in the Fe mass ejected from each generation of SNe II, whereas the amounts of $r$ - and $s$-process elements synthesized in low-mass SNe II and AGB stars, respectively, remain unchanged. As a result, for instance, the $[r$-process $/ \mathrm{Fe}]$ ratio will become higher than that for the case with a normal IMF. Next, we examine the $[\mathrm{Ba} / \mathrm{Fe}]$ ratio in the Fnx GCs at the metallicity of $[\mathrm{Fe} / \mathrm{H}] \sim-2.5$ (upper panel). From their low $[\mathrm{Ba} / \mathrm{Eu}]$ ratios of $\sim-0.7$ (lower panel), Ba contained in the GC stars can be interpreted as a result of the pure $r$-process. Indeed, the high $[r$-process/Fe $]([\mathrm{Ba} / \mathrm{Fe}] \sim 0)$ in the Fnx
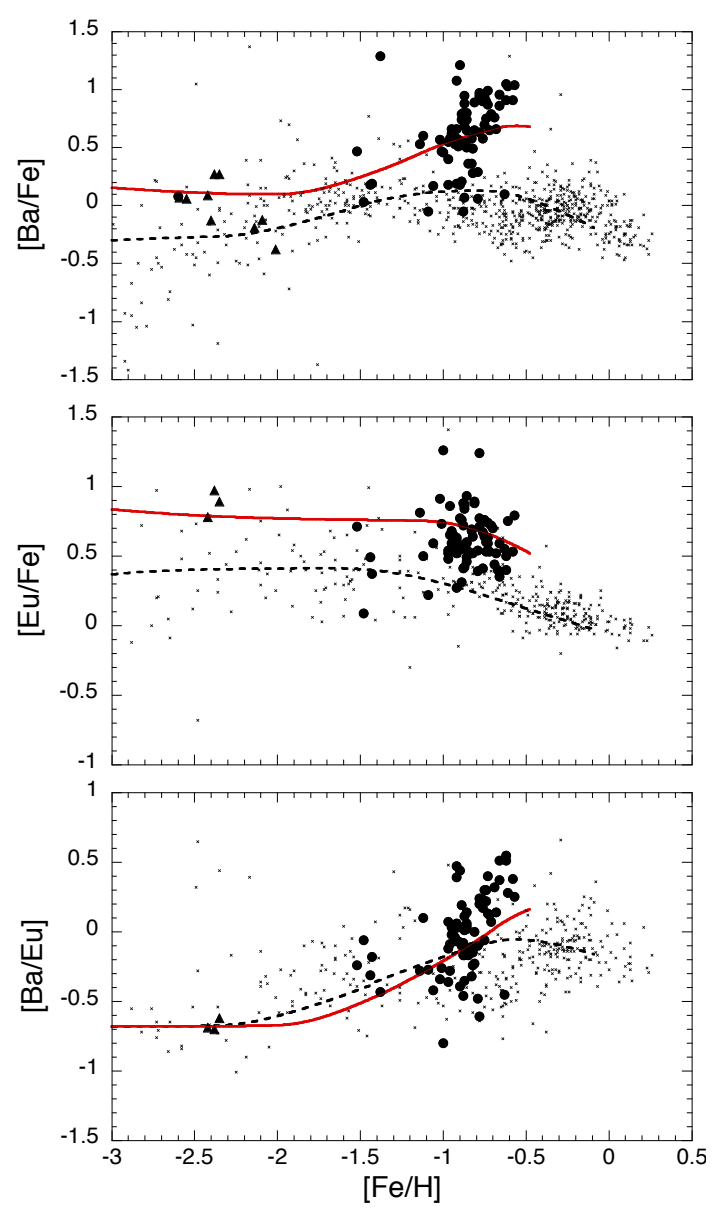

FIG. 1.- Observed and predicted correlations of $[\mathrm{Ba} / \mathrm{Fe}]$ (upper $),[\mathrm{Eu} / \mathrm{Fe}]($ middle $)$, and $[\mathrm{Ba} / \mathrm{Eu}]($ lower $)$ with $[\mathrm{Fe} / \mathrm{H}]$ for the Fnx dSph, compared with those for the Galaxy. The observed data of the Fnx dSph are denoted by filled circles (Letarte et al. 2010), and those for globular clusters are denoted by filled triangles (Letarte et al.|2006). The small crosses show the data for the Galaxy (Venn et al. 2004). The high-s yield for $\mathrm{Ba}$ is assumed in the calculations. Two model curves are obtained for a different set of parameters in which an upper mass end of the IMF plays a principal role, set at $25 M_{\odot}$ (red curve: the Fnx dSph case -model Fnx1-) and $50 M_{\odot}$ (dashed curve: the Galaxy case), respectively.

GCs, compared with Galactic halo stars $(<[\mathrm{Ba} / \mathrm{Fe}]>\sim-$ $0.4)$ is realized. Consistently, high $[\mathrm{Eu} / \mathrm{Fe}]$ ratios are seen in the same GCs as well (middle panel). Further compelling evidence is found in $[r$-process $/ \alpha]$ in the Fnx dSph: our proposed IMF predicts a higher ratio for a whole metallicity range, which is confirmed by the observed high constant $[\mathrm{Eu} / \alpha]$ ratio $(\sim+0.5)$ compared with the Galaxy case $(\sim 0)$ (Letarte et al. 2010). Similarly, after an onset of the enrichment from AGB stars, the $s$-process elements are more predominantly ejected than Fe. This process, together with a stagnant enrichment of Fe that results in the increased ejection of $s$ process elements in the same interval of $\Delta[\mathrm{Fe} / \mathrm{H}]$ as in the Galaxy case, is expected to lead to a sharp increase in $[\mathrm{Ba} / \mathrm{Fe}]$ (or $[\mathrm{Ba} / \mathrm{Eu}])$ against $[\mathrm{Fe} / \mathrm{H}]$, as observed in the Fnx dSph.

\subsection{Model results}


To validate the above processes, we model two cases (the Galaxy and the Fnx dSph) to demonstrate how the different IMFs change the path of chemical evolution between the elements, $\mathrm{Ba}, \mathrm{Eu}$ and $\mathrm{Fe}$. The basis for the model is that the galaxy is formed through an infall of material from outside. With this framework, the SFR is assumed to be proportional to the gas fraction with a constant rate coefficient such as $\nu=0.4 \mathrm{Gyr}^{-1}$ for the Galaxy disk (Tsuijmoto et al. 2010). Here, $\nu$ is the fraction of the gas mass that is converted into stars per Gyr. For the infall rate, we apply a formula that is proportional to $t \exp \left(-t / \tau_{\text {in }}\right)$ with a timescale of infall of $\tau_{\text {in }}$, which is assigned to $\tau_{\text {in }}=5$ Gyr for the Galaxy disk (Yoshii et al. 1996). For the Galactic halo, the values of $\left(\nu, \tau_{\text {in }}\right)=(0.3,0.1)$ are assigned for the duration of star formation $\Delta_{\mathrm{SF}}=0.5 \mathrm{Gyr}$, thus reproducing the abundance distribution function (ADF) of halo stars (Rvan \& Norris 1991). The Galaxy IMF is assumed to be a power-law mass spectrum with a slope of -1.35 , e.g., a Salpeter IMF, with a mass range $\left(m_{l}, m_{u}\right)=\left(0.05 M_{\odot}\right.$, $\left.50 M_{\odot}\right)$ (Tsujimoto et al. 1997). The IMF is always normalized to unity between $m_{l}$ and $m_{u}$, and is combined with the nucleosynthesis yields stated in the following paragraph as well as with the Fe yield of $0.64 M_{\odot}$ from SNe Ia (Iwamoto et al. 1999). It is assumed that the lifetime $t_{\mathrm{Ia}}$ of SN Ia progenitors spans over some range according to a distribution function $g\left(t_{\mathrm{Ia}}\right)$. Here we assume that the fraction $f_{\text {Ia }}$ of the stars that eventually produce $\mathrm{SNe}$ Ia for $3-8 M_{\odot}$ in the solar neighborhood is 0.05 with a box-shaped $g\left(t_{\text {Ia }}\right)$ for $0.5 \leq t_{I a} \leq 3 \mathrm{Gyr}$ (Yoshii et al. 1996). Recent studies on the SN Ia rate in distant galaxies imply the different form of $g\left(t_{\mathrm{Ia}}\right)$ favoring a large population of young progenitors for SNe Ia (Mannucci et al. 2006; Sullivan et al. 2006; Totani et al. 2008; Maoz et al. 2010). However, such a SN Ia frequency distribution is at odds with the presence of an observed plateau of $[\alpha / \mathrm{Fe}]$ among halo stars as well as thick disk stars. Thus, here we adopt the above form so that our model can reproduce the elemental features of the solar neighborhood stars.

Our study highlights the sites and yields of nucleosynthesis, and we consider them for three elements: Fe, $\mathrm{Ba}$ ( $r$ - and $s$-process), and $\mathrm{Eu}$ ( $r$-process). First, our adopted Fe yield increases in accordance with an increase in the mass of the SN II progenitor for $10<M<50$ $M_{\odot}$, which is implied both from the abundance pattern of very metal-poor stars and from an analysis of the SN light curve (Shigevama \& Tsujimoto 1998; Tominaga et al. 2007). As the site for $r$-processes, we adopt the mass range of 20-25 $M_{\odot}$ and the yields for $\mathrm{Ba}$ and Eu (Tsujimoto et al. 2000). We do not have to be exact in the mass range as long as it is set below $\sim 25 M_{\odot}$. This is because its variance influences only the behavior of $r$-process enrichment at a very early epoch, outside the focus of this study. We consider two options for $s$ process yields, each of which provides different timing for the onset of $s$-process enrichment. Observationally, the metallicity indicating this onset among the Galactic halo stars is difficult to detect. It is hidden by the large scatter in the abundance ratios, allowing two claims: either an early start, such as at $[\mathrm{Fe} / \mathrm{H}] \sim-2$ (Gilroy et al. 1988), or a late start, at $[\mathrm{Fe} / \mathrm{H}] \sim-1.4$ (Roederer et al. 2010). Theoretically, the onset of $s$-process enrichment is mainly determined by the $s$-process yield synthesized in low-
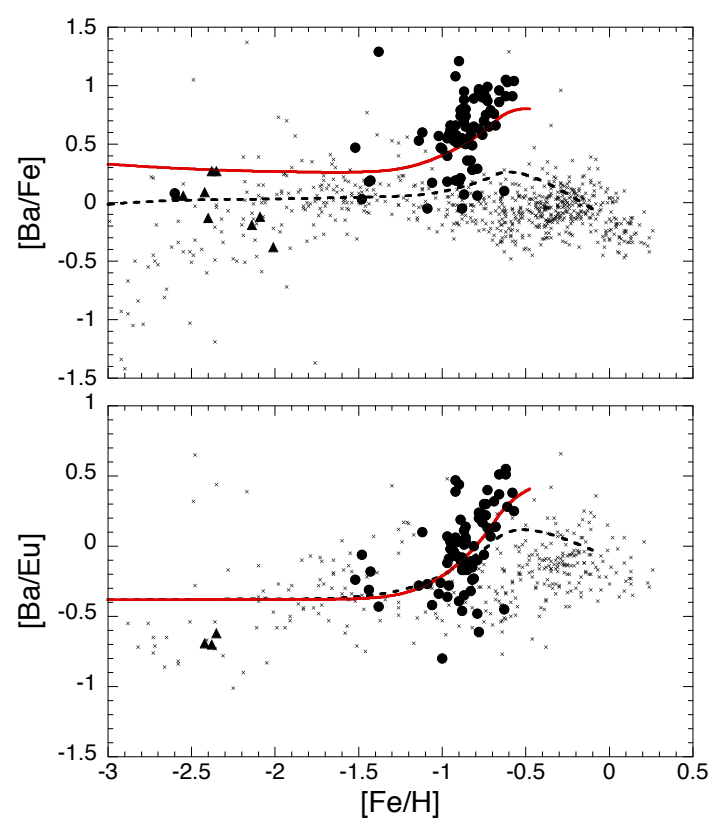

FIG. 2.- The same as in Figure 1 except the model results are with a low- $s$ yield for $\mathrm{Ba}$. The $[\mathrm{Eu} / \mathrm{Fe}]$ vs. $[\mathrm{Fe} / \mathrm{H}]$ plot is not shown since it is the same as the middle panel from Fig. 1.

metallicity stars. If we adopt the results of Busso et al. (2001) having mass range for $s$-process progenitors of 1.5-3 $M_{\odot}$, their very low- $s$ yield for low-metallicity AGB stars results in basically no contribution of the $s$-process to halo stars (see Cescutti et al. 2006), and thus realizes a late start. We refer to this option as the low- $s$ yield case. On the other hand, to enable an early start, i.e., an onset of the influence of the $s$-process on very lowmetallicity stars, we prepare another option for the $s$ process yield by diminishing the metallicity dependency for a low-metallicity regime in the above low- $s$ yield case. In this high- $s$ yield case, the wider mass range of 1.5-6 $M_{\odot}$, including hot AGB stars, is adopted.

Figure 1 shows the results calculated with the high- $s$ yield, with the Galaxy models denoted by the dashed curves. Due to the high- $s$ yield, the $[\mathrm{Ba} / \mathrm{Fe}]$ ratio gradually starts to increase from $[\mathrm{Fe} / \mathrm{H}] \sim-2.2$. After that the increase is suppressed due to a contribution of $\mathrm{Fe}$ from $\mathrm{SNe} \mathrm{Ia}$, and then the ratio decreases from $[\mathrm{Fe} / \mathrm{H}] \sim-0.7$, which is caused by a decreasing $s$-process Ba yield with the metallicity (upper panel). A different trend is apparent in the evolution of $[\mathrm{Eu} / \mathrm{Fe}]$, which is simply "a plateau plus a decreasing phase" in the same manner as the $[\alpha / \mathrm{Fe}]$ evolution. This trend is attributed to a negligible $s$-process production of $\mathrm{Eu}$ (middle panel). These two results lead to the $[\mathrm{Ba} / \mathrm{Eu}]$ evolution as shown in the lower panel.

Next we examine the models for the Fnx dSph, to determine if an increase in $[\mathrm{Ba} / \mathrm{Fe}]$ at a late evolution can be reproduced. As discussed in the previous sections, this mechanism requires the introduction of a smaller $m_{u}$ for the IMF, set at $m_{u}=25 M_{\odot}$. In addition another setting is required for the model; that is, a relatively efficient star formation is demanded in order to prevent an increase in $[\mathrm{Ba} / \mathrm{Fe}]$ from occurring at a lower metallicity than ob- 
served. This view is compatible with the observed ADF of stars that are older than $10 \mathrm{Gyr}$, which extends to the metal-rich end around $[\mathrm{Fe} / \mathrm{H}]=-0.5$ (Coleman \& de Jong 2008). Here, $\nu=2 \mathrm{Gyr}^{-1}$ and $\tau_{\text {in }}=0.1 \mathrm{Gyr}$ are chosen with $\Delta_{\mathrm{SF}}=1.5$ Gyr. The fraction of SNe Ia is determined to be $f_{\mathrm{Ia}}=0.03$ by the late evolution of $\mathrm{Ba}$ and Eu against Fe. We refer to this model as model Fnx1, since we will prepare for another two models in order to understand the chemical evolution of the Fnx dSph in $\S 3$. The results are indicated by the red curves in each panel. Each predicted curve passes through the GC data and broadly fits the observed trend at a late phase for $-1.5 \lesssim[\mathrm{Fe} / \mathrm{H}] \lesssim-0.5$.

Results for the low- $s$ yield case are shown in Figure 2. This case requires the assumption of a high $[\mathrm{Ba} / \mathrm{Fe}]$ ratio $(\sim 0)$ as a plateau value for the halo, by raising the integrated $r$-process Ba yield by a factor of $\sim 2$. According to this change, to suppress the increase in $[\mathrm{Ba} / \mathrm{Fe}]$ at a very low metallicity for the Fnx dSph case, we reduce the $r$-process Ba yield by a factor of 0.6, compared to the $r$-process yield in the Galaxy case. These modifications are based on the hypothesis that the Ba yield for the mass $M \leq 25 M_{\odot}$ is broadly the same as in the high- $s$ yield case while the rest is synthesized in SNe II with $M>25 M_{\odot}$. Again we see good agreement of the model result assuming a small $m_{u}$ with the observed increasing Ba feature.

\subsection{Insight into $Y$ nucleosynthesis site}

Our study will be put forward to the examination into the nucleosynthesis of some other elements in the framework that connects their elemental features to our proposed view. Here we discuss the nucleosynthesis site of the light $s$-process element Y. Regarding $\alpha$-elements, detailed discussion will be presented in $\S 4$.

Yttrium $(A=89)$ has been well observed in dSphs (e.g., Shetrone et al. 2001), including the Fnx dSph (Letarte et al. 2010). In regard to the $s$-process for the light $s$-elements with mass number $70 \lesssim A \lesssim 90$, the core He-burning in massive stars, the so-called weak $s$ process, is considered to be more important than the $s$-process operating in AGB stars. The weak $s$-process yield is found to increase with stellar mass (Pumo et al. 2010). Moreover, a recent study claims that the $r$-process for $A \sim 90-110$ is produced by a charged particle reaction (CPR) and shares the same site with Fe production in massive stars (Qian \& Wasserburg 2007). These theoretical results, together with the additional assumption of a similar mass-dependence between the $r$-process $\mathrm{Y}$ and Fe yields, provide a consistent interpretation of the $\mathrm{Y}$ feature present in the middle and lower panels. There is a broad coincidence of the $[\mathrm{Y} / \mathrm{Fe}]$ value at the same $[\mathrm{Fe} / \mathrm{H}]$ between the Galaxy and the Fnx dSph over an entire metallicity range, while there is a large deviation of $[\mathrm{Ba} / \mathrm{Y}]$ between two galaxies in the late evolution. In Figure 3, the model results for the two galaxies are superimposed. Here we adopt the $\mathrm{Y}$ yields which have a mass-dependence similar to Fe in massive stars for both $r$ - and $s$ - processes, and ignore the AGB contribution for $\mathrm{Y}$.

\section{CHEMICAL EVOLUTION OF THE FORNAX DSPH} GALAXY

The model presented in $\S 2.2$ (i.e., model Fnx1) that reproduces the observed $\mathrm{Ba}$ feature is equipped with a

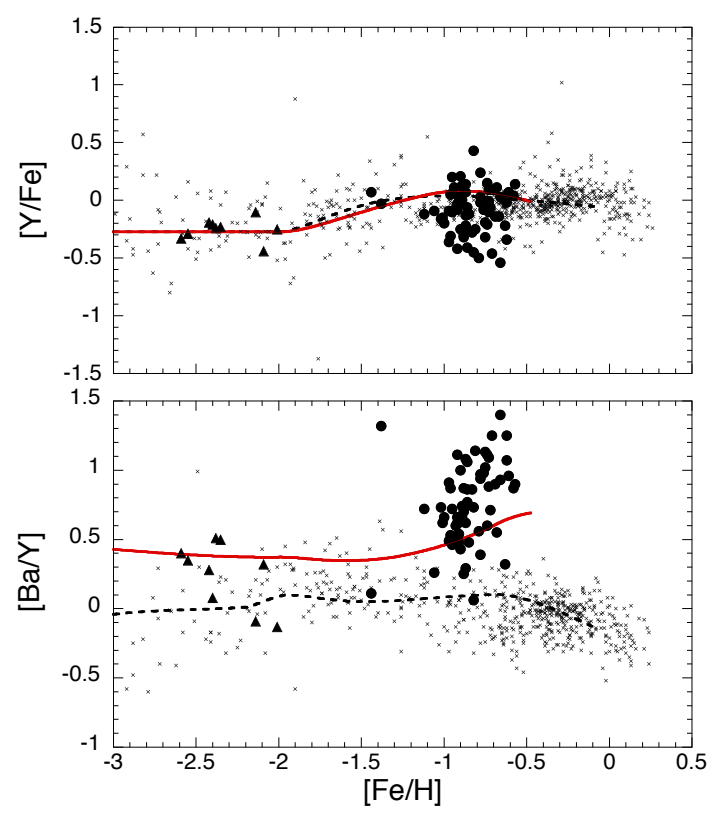

FIG. 3.- Observed and predicted correlations of $[\mathrm{Y} / \mathrm{Fe}]$ and $[\mathrm{Ba} / \mathrm{Y}]$ with $[\mathrm{Fe} / \mathrm{H}]$ for the Fnx dSph (red curve), compared with those for the Galaxy (dashed curve).

rather short timescale $(=1.5 \mathrm{Gyr}$ ) for star formation. Such rapid enrichment is observationally supported by the presence of old ( $>10$ Gyr) stars having metallicity up to $[\mathrm{Fe} / \mathrm{H}] \sim-0.5$ (Coleman \& de Jong 2008). On the other hand, the Fnx dSph has a much longer star formation history on the whole (Battaglia et al. 2006; Coleman \& de Jong 2008). These two aspects seem to require the introduction of the complex model into the framework to consistently explain both the elemental feature and the overall star formation history. As a likely case, we can raise the possibility that different paths of chemical enrichment exist leading to a large variation in the abundances of the present-day Fnx dSh stars. It is, at least in part, inclined to result from the different speeds of star formation depending on the distance from the galaxy center, that will appear as an observed radial metallicity gradient (Battaglia et al. 2006; Coleman \& de Jong 2008).

In fact, a close look at the $[\mathrm{Ba} / \mathrm{Fe}]$ evolution in Figure 1 shows that some stars in the Fnx dSph are located right on the predicted path for the Galaxy. This implied channel is modeled by $\left(\nu, \Delta_{\mathrm{SF}}\right)=(0.08,5)$ with a normal IMF, i.e., $m_{u}=50 M_{\odot}$. This case is referred to as model Fnx2. In addition, the presence of the age-metallicity relation in the Fnx dSph (Battaglia et al. 2006; Coleman \& de Jong 2008) implies that a large proportion of the metal-rich stars leading to an increasing $\mathrm{Ba} / \mathrm{Fe}$ trend are relatively young. Incorporating the observed finding of an enhanced star formation at a very late epoch (Coleman \& de Jong 2008) into the model, we set $\left(\nu, \Delta_{\mathrm{SF}}\right)=(1,2)$ with $m_{u}=25 M_{\odot}$ and an initial metallicity of $[\mathrm{Fe} / \mathrm{H}]=-1.2$ for this case, and refer to it as model Fnx3. Then, together with the first model presented in $\S 2.2$, i.e., model Fnx1, the Ba/Fe evolutions predicted by the three models in total are shown 

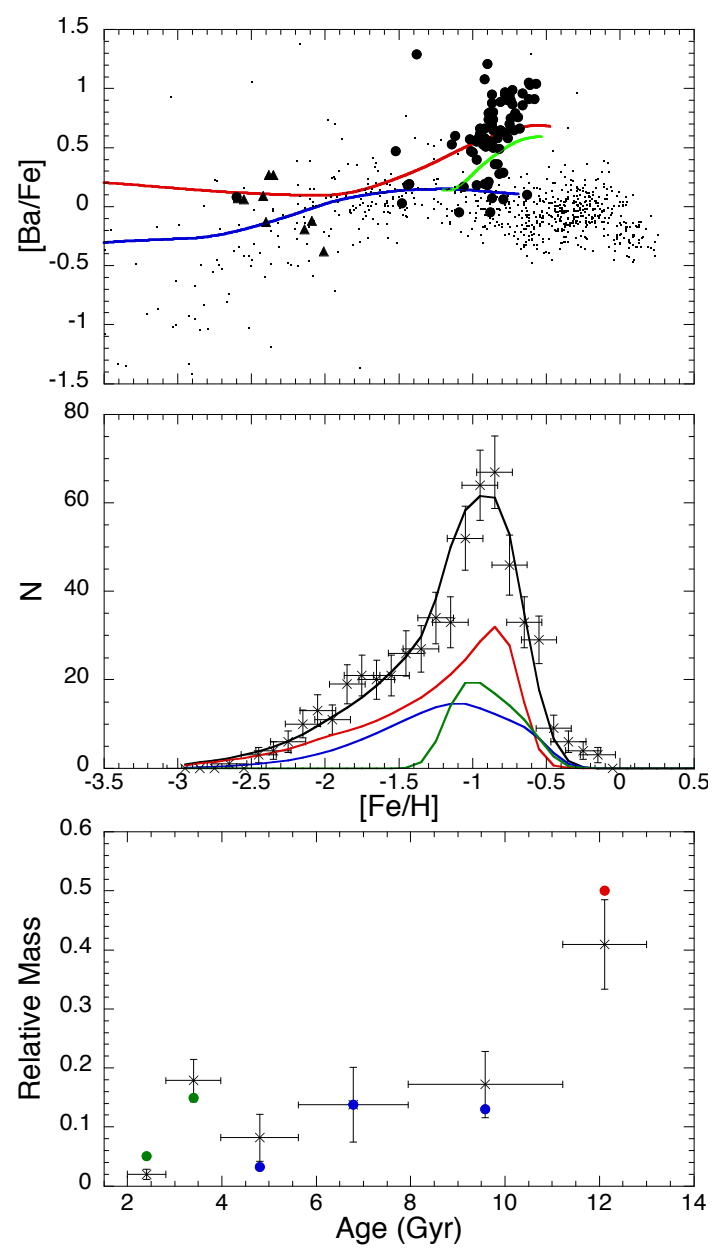

FIG. 4.- Predicted chemical features of the Fnx dSph superimposed by three different paths of chemical enrichment, compared with observations. Upper panel: The $[\mathrm{Ba} / \mathrm{Fe}]$ vs. $[\mathrm{Fe} / \mathrm{H}]$. Each curve with a different color corresponds to a different calculated path (red curve: model Fnx1, blue curve: model Fnx2, green curve: model Fnx3). Middle panel: Predicted ADF of the Fnx $\mathrm{dSph}$ stars against $[\mathrm{Fe} / \mathrm{H}]$. The calculated distribution is summed up by those by the individual model distributions, with a ratio of 0.5 (model Fnx1)/0.3 (model Fnx2)/0.2 (model Fnx3), and is convolved using the Gaussian with a dispersion of 0.1 dex considering a measurement error expected in the data. Each contribution by three models is shown by a different color. Crosses represent data taken from Battaglia et al. (2006). Lower panel: Predicted relative stellar mass formed during each age bin (filled circles), compared with the observed results (crosses: Coleman \& de Jong 2008).

in the upper panel of Figure 4. In the middle panel, the resultant ADF summed up by the individual models with a ratio of 0.5/0.3/0.2 is compared with the observation (Battaglia et al. 2006). The star formation history constructed by these relative contributions from individual models is compared with the observed history (Coleman \& de Jong 2008), which is reassessed without counting very low star formation for $t<2 \mathrm{Gyr}$ and an age threshold of $13 \mathrm{Gyr}$, in the lower panel.

Here we review the adopted models in the context of the integrated galactic IMF (IGIMF) theory (Kroupa \& Weidner 2003; Weidner \& Kroupa 2005). The IGIMF predicts that $m_{u}$ depends on the SFR (or gas mass) in a sense that $m_{u}$ is smaller according to a lower SFR. Thus, our hypothesis that model Fnx2 with a moderate SFR is equipped with a larger $m_{u}$ than models Fnx1\&3 with a higher SFR seems at odds with the IGIMF theory. As one possible explanation to integrate our models into this theoretical scheme, the stellar population associated with model Fnx2 may be regarded as that originally belonging to more massive building block within a hierarchical galaxy formation scenario. That casts our attention on the observed fact that the Fnx dSph obeys the luminosity-metallicity relation (Kirby et al. 2008; Revaz et al. 2009). This property may provide the possible path to the complex populations such that the merger event of Fnx2 into Fnx1 induces the enhanced star formation, and yields a metalrich component Fnx3, then ultimately ends up with a metallicity increase as a whole in accordance with the mass growth of galaxy. On the other hand, a maximal IGIMF model together with the total mass of $1.9 \times 10^{7} M_{\odot}$ for the Fnx dSph (Woo te al. 2008) approximately gives $m_{u}=30,20 M_{\odot}$ to model Fnx1, Fnx3, respectively. In this study, we have a close look at the signature of a small $m_{u}$ at an early evolutionary stage. However, given that we ignore it, one standard chemical evolution model that predicts a declining gas mass (SFR) as a function of elapsed time (Kirby et al. 2011) is expected to lead to a gradual reduction in $m_{u}$ that will make the $\mathrm{Ba} / \mathrm{Fe}$ ratio mildly upward during late evolution.

\section{UNDERSTANDING OF THE $\alpha /$ FE FEATURE}

Finally, we examine the $\alpha / F e$ feature in the framework of the models constructed in the previous section. The prediction of the ratio of $\mathrm{O}$ ( or $\mathrm{Mg}$ ) to $\mathrm{Fe}$ ejected from an individual SN II still involves uncertainties because these two elements are synthesized by a completely different process. $\mathrm{O}(\mathrm{Mg})$ is produced through hydrostatic nuclear burning during stellar evolution before the SN explosion, whereas the amount of Fe is determined by the explosion mechanism and fallback dynamics, which are not fully understood yet. However, the observed feature of $[\alpha / \mathrm{Fe}]$ in $\mathrm{dSphs}$ cannot be fully interpreted by the knee structure positioned at a low-metallicity, since there exist stars exhibiting low $[\alpha / \mathrm{Fe}]$ ratios at a very low $[\mathrm{Fe} / \mathrm{H}]$ (see the distinct case of the Sextan dSph; Aoki et al. 2009).

Suppose that the average $\alpha / \mathrm{Fe}$ ratio integrated over 10-25 $M_{\odot}$ becomes small with the assumption that an increasing trend of yield against the progenitor mass is more prominent for $\alpha$-elements than for Fe. Here we assume that a truncated IMF in models Fnx1 and Fnx3 invokes that the average $\mathrm{O}, \mathrm{Mg}$, and $\mathrm{Ti}$ yields from $\mathrm{SNe}$ II are reduced by a factor of $0.7 / 0.5 / 0.3$. Together with model Fnx 2, the results predicted by the three models for the Fnx dSph are shown by the colored curves, while the dashed curves represent the Milky Way case. For the nucleosynthesis yields of $\mathrm{O}$ and $\mathrm{Mg}$ form $\mathrm{SNe}$ II, we take their values tabulated in Tsujimoto et al. (1995). On the other hand, the Ti yield as a function of progenitor's mass is deduced from the abundance analysis of very metal-poor stars as performed by Tsujimoto \& Shigevama (1998), because the existing nucleosynthesis models fail to predict the Ti yield which is constrained form the observed level of $[\mathrm{Ti} / \mathrm{Fe}]$ among halo stars (see Kobavashi et al. 2006). For [O/Fe] and 

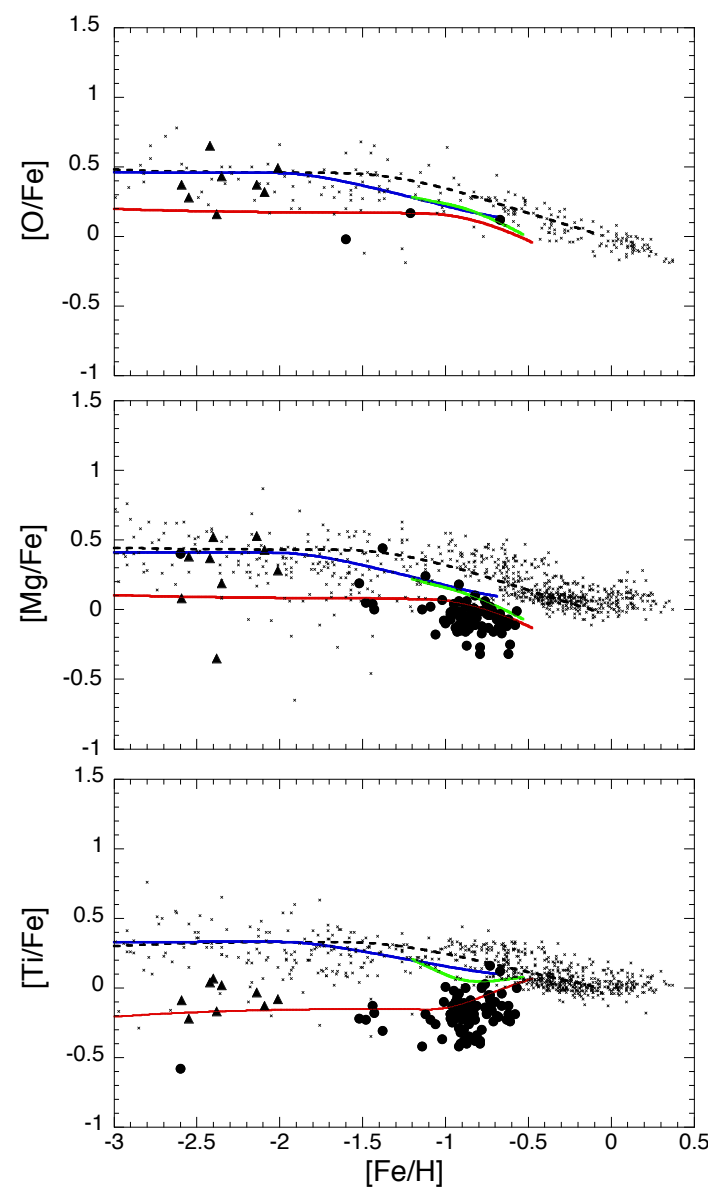

FIG. 5.- Observed and predicted correlations of $[\mathrm{O} / \mathrm{Fe}]$ (upper), $[\mathrm{Mg} / \mathrm{Fe}]($ middle) , and $[\mathrm{Ti} / \mathrm{Fe}]($ lower $)$ with $[\mathrm{Fe} / \mathrm{H}]$ for the Fnx dSph, together with those for the Galaxy. Each curve with a different color corresponds to a different calculated path for the Fnx dSph (red curve: model Fnx1, blue curve: model Fnx2, green curve: model Fnx3), while the dashed curve denotes the Galaxy case. For models Fnx1 and Fnx3, the modified nucleosynthesis SN II yields of $\alpha$-elements are adopted (see the text). Symbols are the same as in Figure 1 except for $[\mathrm{O} / \mathrm{Fe}]$ (filled circles: Shetrone et al. [2003, crosses: Edvardsson et al. 1993; Gratton et al. 2000; Cayrel et al. 2004; Bensby et al. 2005).

$[\mathrm{Mg} / \mathrm{Fe}]$, the observed properties, i.e., a large scatter at a low-metallicity and an overall decreasing trend, are well reproduced. For $[\mathrm{Ti} / \mathrm{Fe}]$, the observed data around $[\mathrm{Ti} / \mathrm{Fe}] \sim+0.3$ is lacking. However, medium-resolution observations suggest the presence of stars exhibiting such a high ratio (Kirby et al. 2010). Note that an unusual upward $\mathrm{Ti} / \mathrm{Fe}$ feature for $[\mathrm{Fe} / \mathrm{H}] \gtrsim-1$ is reproduced by the combination of low and high $\mathrm{Ti} / \mathrm{Fe}$ yields in $\mathrm{SNe}$ II and $\mathrm{SNe} \mathrm{Ia}$, respectively. Since $\mathrm{Ca}$ is also synthesized highly in SNe Ia, our model predicts a similar upward feature for $\mathrm{Ca} / \mathrm{Fe}$. This is indeed observed in the $\mathrm{Fnx}$ dSph (Letarte et al. 2010).

Our model anticipates that the observed dispersion in both $[\mathrm{Ba} / \mathrm{Fe}]$ and $[\mathrm{Mg} / \mathrm{Fe}]$ at a low-metallicity is an end result of the IMF variation which yields a high $[\mathrm{Ba} / \mathrm{Fe}]$ and a low $[\mathrm{Mg} / \mathrm{Fe}]$ by $m_{u}=25 M_{\odot}$ and a reverse correlation by $m_{u}=50 M_{\odot}$. Figure 6 demonstrates that the predicted correlation of $[\mathrm{Ba} / \mathrm{Fe}]$ and $[\mathrm{Mg} / \mathrm{Fe}]$ is broadly

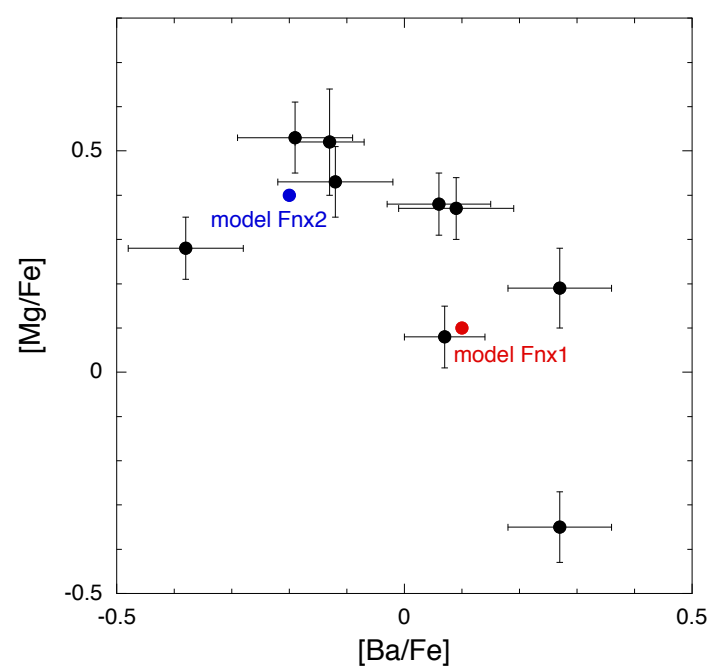

FIG. 6. - The observed correlation of $[\mathrm{Ba} / \mathrm{Fe}]$ and $[\mathrm{Mg} / \mathrm{Fe}]$ for the GC in the Fnx dSph compared with those predicted by model Fnx1 $\left(m_{u}=25 M_{\odot}\right)$ and model Fnx2 $\left(m_{u}=50 M_{\odot}\right)$ at $[\mathrm{Fe} / \mathrm{H}] \approx-2.5$.

compatible with the observed one given by the data of the Fnx GC, though this analysis should be validated by much more data.

\section{DISCUSSION AND CONCLUSIONS}

Observed unusual $\mathrm{Ba}$ enhancement relative to $\mathrm{Fe}, \alpha$ elements, and Eu in some dSphs as well as in the LMC at their late evolution is investigated by modeling the Fornax dSph galaxy case. Our claim is that its effect occurs because the IMFs truncate a high mass end at around $25 M_{\odot}$, that causes the reduction of $\alpha$-elements and $\mathrm{Fe}$ but no (little) influence on $r$ - or $s$-process elements in their ejection, associated with the death of stars covering a wide range of masses. Such a truncated IMF is assured by the theoretical agument given by the IGIMF scheme in which the number of massive stars depends on the SFR in galaxies. In addition, the star formation history of the Fnx dSph revealed by recent surveys, together with a large dispersion in elemental ratios such as $[\alpha / \mathrm{Fe}]$ and $[\mathrm{Ba} / \mathrm{Fe}]$, suggests a rather complex chemical history not represented by a single model but composed of a few evolutionary paths resulting from a different speed of star formation as well as from a different form of the IMF.

Previous work on the chemical evolution of dSphs interpret the lower $[\alpha / \mathrm{Fe}]$ ratios in these galaxies as being due to a combination of the time-delay model and a low SFR (e.g., Carigi et al. 2002; Ikuta \& Arimoto 2002; Lanfranchi \& Matteucci 2003; Kirby et al. 2011). This interpretation can explain the observed $[\mathrm{Ba} / \mathrm{Eu}]$ ratios in dSphs with the inclusion of the galactic wind effect (Lanfranchi et al. 2008); however, it does not favor the same increasing $[\mathrm{Ba} / \mathrm{Fe}]$ trend as $[\mathrm{Ba} / \mathrm{Eu}]$ (see, however, Fenner et al. 2006, as the model with a different assumption on galactic wind for the Sculptor dSph). Further, it confronts the fact that a massive dwarf galaxy, i.e., the LMC, exhibits the same level of increase in $[\mathrm{Ba} / \mathrm{Fe}]$ as would the much smaller Fnx dSph. On the other hand, our proposed model has the following shortcomings: (1) the lower $[\alpha / \mathrm{Fe}]$ is shifted to the uncertainties in theoret- 
ical nucleosynthesis in massive stars, (2) we lack an explanation for what the major driver of a complex star formation/chemical enrichment history is in the Fnx dSph, (3) we lack a sufficient explanation for why and how the high mass end of the IMF varies inside the Fnx dSph in the context of the IGIMF thoery. These issues point to the need for future studies.
The author wishes to thank an anonymous referee for his/her valuable comments and excellent review, that has considerably improved the paper, and is assisted in part by Grant-in-Aid for Scientific Research (21540246) of the Japanese Ministry of Education, Culture, Sports, Science and Technology.
Aoki, W., et al. 2009, A\&A, 502, 569

Battaglia, G., et al. 2006, A\&A, 459, 423

Bensby, T., Feltzing, S., Lundström, I., \& Ilyiin, I. 2005, A\&A, 433, 185

Busso, M., Gallino, R., Lambert, D. L., Travaglio, C., \& Smith, V. V. 2001, ApJ, 557, 802

Carigi, L., Hernandez, X., \& Gilmore, G. 2002, MNRAS, 334, 117 Cayrel, R., et al. 2004, A\&A, 416, 1117

Cescutti, G., François, P., Matteucci, F., Cayrel, R., \& Spite, M. 2006, A\&A, 448, 557

Cohen, J. G., \& Huang, W. 2009, ApJ, 701, 1053

Coleman, M. G., \& de Jong, J. T. A. 2008, ApJ, 685, 933

Edvardsson, B., Andersen, J., Gustaffson, B., Lambert, D. L., Nissen, P. E., \& Tomkin, J. 1993, A\&A, 275, 101

Fenner, Y., Gibson, B. K., Gallino, R., \& Lugaro, M. 2006, ApJ, 646,184

Frebel, A., Kirby, E. N., \& Simon, J. D. 2010a, Nature, 464, 72

Frebel, A., Simon, J. D., Geha, M., \& Willman, B. 2010b, ApJ, 708,560

Freeman, K., \& Bland-Hawthorn, J. 2002, ARA\&A, 40, 487

Gilroy, K. K., Sneden, C., Pilachowski, C. A., \& Cowan, J. J. 1988, ApJ, 327, 298

Goriely, S., \& Siess, L. 2004, A\&A, 421, L25

Gratton, R. G., Sneden, C., Carretta, E., \& Bragaglia, A. 2000 A\&A, 354,169

Hill, V. 2004, in Carnegie Observatories Astrophysics Series, Vol. 4, Origin and Evolution of the Elements, ed. A. McWilliam \& M. Rauch (Cambridge: Cambridge Univ. Press), 203

Ikuta, C., \& Arimoto, N. 2002, A\&A, 391, 55

Ishimaru, Y., \& Wanajo, S. 1999, ApJ, 511, L33

Iwamoto, K., et al. 1999, ApJS, 125, 439

Kirby, W. N., Simon, J. D., Geha, M., Guhathakurta, P., \& Frebel A. 2008 , ApJ, $685, \mathrm{~L} 43$

Kirby, E. N., et al. 2010, ApJS, 191, 352

Kirby, E. N., Cohen, J. G., Smith, G. H., Majewski, S. R., Sohn, S. T., \& Guhathakurta, P. 2011, ApJ, 727, 79

Kobayashi, C., Umeda, H., Nomoto, K., Tominaga, N., \& Ohkubo, T. 2006, ApJ, 653, 1145

Koch, A., et al. 2008, AJ, 135, 1580

Kroupa, P., \& Weidner, C. 2003, ApJ, 598, 1076

Lanfranchi, G. A., \& Matteucci, F. 2003, MNRAS, 345, 71

Lanfranchi, G. A., Matteucci, F., \& Cescutti, G. 2008, A\&A, 481, 635

Lee, J. C. et al. 2009, ApJ, 706, 599

Letarte, B., Hill, V., Jablonka, P., Tolstoy, E., François, \& Meylan, G. $2006, A \& A, 453,547$

Letarte, B., et al. 2010, A\&A, 523, 17

\section{EFERENCES}

Mannucci, F., Della Valle, M., \& Panagia, N. 2006, MNRAS, 370, 773

Maoz, D., Sharon, K., \& Gal-Yam, A. 2010, ApJ, 722, 1879

Mathews, G. J., Bazan, G., \& Cowan, J. J. 1992, ApJ, 391, 719

Meurer, G. R., et al. 2009, ApJ, 695, 765

Pagel, B. E. J. 1997, Nucleosynthesis and Chemical Evolution of Galaxies (Cambridge: Cambridge Univ. Press)

Pflamm-Altenburg, J., \& Kroupa, P. 2008, Nature, 395, 394

Pompéia, L., et al. 2008, A\&A, 480, 379

Pumo, M. L., Contino, G., Bonanno, A., \& Zappalá, R. A. 2010, A\&A, 524,45

Qian, Y.-Z., \& Wasserburg, G. J. 2007, Phys. Rep., 444, 237

Revaz, Y, et al. 2009, A\&A, 501, 189

Roederer, I. U., et al. 2010, ApJ, 724, 975

Ryan, S. G., \& Norris, J. E. 1991, AJ, 101, 1865

Shetrone, M., Côté, P., \& Sargent, W. L. W. 2001, ApJ, 548, 592

Shetrone, M., Venn, K. A., Tolstoy, E., Primas, F., Hill, V., \& Kaufer, A. 2003, AJ, 125, 684

Shigeyama, T., \& Tsujimoto, T. 1998, ApJ, 507, L135

Sbordone, L., Bonifacio, P., Buonanno, R., Marconi, G., Monaco, L., \& Zaggia, S. 2007, A\&A, 465, 815

Sullivan, M. et al. 2006, ApJ, 648, 868

Tolstoy, E., Hill, V., \& Tosi, M. 2009, ARA\&A, 47, 371

Tominaga, N., Umeda, H., \& Nomoto, K. 2007, ApJ, 660, 516

Totani, T., Morokuma, T., Oda, T., Doi, M., \& Yasuda, N. 2008, PAS.J, 60,1327

Tsujimoto, T. 2006, A\&A, 447, 81

Tsujimoto, T., Nomoto, K., Yoshii, Y., Hashimoto, M., Yanagida, S., \& Thielemann, F.-K. 1995, MNRAS, 277, 945

Tsujimoto T., Yoshii Y., Nomoto K., Matteucci F., Thielemann F.-K., Hashimoto M., 1997, ApJ, 483, 228

Tsujimoto, T., \& Shigeyama, T. 1998, ApJ, 508, L151

Tsujimoto, T., Shigeyama, T., \& Yoshii, Y. 2000, ApJ, 531, L33

Tsujimoto T., Bland-Hawthorn J., Freeman K. C., 2010, PASJ, 62, 447

Tsujimoto, T., \& Bekki, K. 2011, A\&A, 530, A78

Yoshii, Y., Tsujimoto, T., \& Nomoto, K. 1996, ApJ, 462, 266

Wheeler, J. C., Cowan, J. J., \& Hillebrandt, W. 1998, ApJ, 493 101

Weidner, C., \& Kroupa, P. 2005, ApJ, 625, 754

Woo, J., Courteau, S., \& Dekel, A. 2008, MNRAS, 390, 1453

Venn, K. A., Irwin, M., Shetrone, M. D., Tout, C. A., Hill, V., \& Tolstoy, E. 2004, AJ, 128, 1177

Venn, K. A., \& Hill, V. 2008, The Messenger, Supplement Series, 134,27 